\title{
The studies on waste biodegradation by Tenebrio molitor
}

\author{
Magdalena Bożek ${ }^{1}$, Beata Hanus-Lorenz ${ }^{1}$, and Justyna Rybak $^{1,{ }^{*}}$ \\ ${ }^{1}$ Wrocław University of Science and Technology, Department of Environmental Engineering, \\ Wybrzeże Wyspiańskiego 27, 50-370 Wrocław,
}

\begin{abstract}
As cities are growing in size with a rise in the population, the amount of plastic waste generated is increasing and becoming unmanageable. The treatment and disposal of plastic waste is an urgent need of our present and future. It has been proved recently that mealworms, the larvae of Tenebrio molitor Linnaeus, are able eat styrofoam, a common polystyrene product. Polystyrene is one of the most widely used plastics, the scale of its production being several million tons per year. Tenebrio molitor is one of the largest pests found in stored-grain products. The insect is indigenous to Europe, but is currently cosmopolitan in distribution. The styrofoam is efficiently degraded in the larval gut by microorganisms. We have used the larvae of $T$. molitor to biodegrade three types of food packaging plastics: polystyrene (PS), polyvinyl chloride (PVC) and polylactide (PLA). PVC is a thermoplastic made of $57 \%$ chlorine (derived from industrial grade salt) and $43 \%$ carbon (derived predominantly from oil /gas via ethylene). It is the world's third-most widely produced synthetic plastic polymer, which is not biodegradable easily. On the other hand, PLA is an easily biodegradable and bioactive thermoplastic aliphatic polyester derived from corn and tapioca starch or sugarcane. Three groups of larvae were fed selected types of polymers as an only food, while a control population was fed on oatmeal. The mass loss, dry matter content and biochemical composition of mealworms were assessed in the performed laboratory experiments. The protein concentration in homogenates of the larvae was determined by the Bradford method. To determine the level of hydrolized carbohydrates we used anthrone method. The classical sulfo-phospho-vanillin assay (SPVA) was used to quantitate total lipids in mealworms. The results allowed to compare the decomposition efficiency of selected polymer materials by mealworms and to recognize the mechanism of decomposition contributing to the future use of these animals for the treatment and disposal of plastic waste.
\end{abstract}

\section{Introduction}

One of the most persistent pollutants which are very resistant to biodegradation in the environment is plastics. Their accumulation in terrestrial habitats can be dangerous as

* Corresponding author: justyna.rybak@pwr.edu.pl 
plastics pose the serious threat to the environment health. Plastics can be degraded by chemical, thermal, photo or biological degradation [1].

Polystyrene (PS) is one of the most difficult material for biodegradation. There are only few examples of its biodegradation, but at the same time it is commonly used and widespread. The production of PS reaches several million tons per year [2]. In the recent studies scientists have been proved that styrofoam can be degraded effectively by bacteria which were discovered in the gut of larvae of Tenebrio molitor Linnaeus [3]. This is the unique evidence that mealworms can be employed in the biodegradation of plastics.

Polyvinylchloride (PVC) is a very strong material, resistant to abrasion PVC consisting of $57 \%$ chlorine (from industrial grade salt) and $43 \%$ carbon (from oil /gas via ethylene). It is commonly used in pipes, hoses and floor coverings. PVC can be decomposed by chemical and phytodegradation but degradation with the use of bacteria have been observed extremely rare [1]. The experiments concerning the biodegradation of PVC with bacteria which are present in the gut of $T$. molitor have never been performed.

Thus, the aim of our studies was to compare the efficiency of biodegradation of two plastics which belong to the most resistant and almost non-biodegradable materials. The mealworms were employed for the waste biodegradation. As a comparison the third material, polylactide (PLA), was used. PLA is a natural, biodegradable plastic, made from renewable resources (such as starch, corn or sugarcane) which can be naturally produced or synthesized from renewable resources [4]. This is a sustainable alternative to nonrenewable petroleum- based synthetic biodegradable plastics. The lactides present in this material can be easily produced by the microorganisms in the fermentation process of agricultural by-products, mainly the carbohydrate rich substances [4]. The mealworms were fed these three materials (PS, PVC, PLA). Here, we report evidence that biodegradation of PS and PVC in comparison to PLA and observation made for control insects does occur. This observation was based on the mass loss of studied waste. What is more, feeding on these food sources can theoretically bring energy to mealworms, thus the effect on lipid, total sugar and carbohydrates contents of larvae was also examined under laboratory conditions. Additonally, we also studied the starved mealworms as a comparison to larvae fed biodegradable and non-biodegradable plastics.

\section{Materials and methods}

\subsection{Materials}

Tenebrio molitor is a species of darkling beetles which belongs to Tenebrionidae family. Like all holometabolic insects they undergo four life stages: egg, larva, pupa and imago. Larvae measure about $2.5 \mathrm{~cm}$, whereas adults have $1.25-1.8 \mathrm{~cm}$ length. Adults individuals lay eggs in the soft ground and then die. The optimum temperature for breeding is within the range of $25-35^{\circ} \mathrm{C}$. In natural conditions, beetles are found in decaying and rotting bark of deciduous trees. The larvae and adult beetles feed on dead organisms and live in warehouses cereal products, pantries, poultry farms and dovecotes. In this experiment larvae of T. molitor came from the inbreeding farm in Lublin (Poland).

\subsection{Methods}

Experiment was carried out in the parallel 5 groups $(20$ specimens each, the parameter for selecting 20 specimens in each group was their similar mass). Larvae were weighted weekly. The variants used in tests were as follows:

- larvae eating oatmeal ad libitum (control); 
- larvae eating polystyrene (PS) in the form of $1 \mathrm{~mm}$ thick plates manufactured in the laboratory (raw material);

- larvae eating polylactide (PLA) in the form of $1 \mathrm{~mm}$ thick plates manufactured in the laboratory (raw material);

- larvae eating polyvinyl chloride (PVC) for medical purposes, as commercially available tubing for oxygen supply cut in $10 \mathrm{~mm}$ pieces;

- starved larvae.

After 3 weeks larvae were homogenized using cold mortar and pestle with ice-cold $0.1 \mathrm{M}$ phosphate buffer $\mathrm{pH} 7.4,1 \mathrm{mM}$ EDTA $(1: 10 \mathrm{w} / \mathrm{v})$. For carbohydrates assay aliquots of homogenates were frozen directly for further analysis. Required aliquots for lipid assay were treated with chloroform: methanol $(1: 2 \mathrm{v} / \mathrm{v})$. The rest of homogenate were centrifuged at $11000 \mathrm{x} \mathrm{g}, 5 \mathrm{~min}$ and frozen in aliquots at $-20^{\circ} \mathrm{C}$ in $2 \mathrm{ml}$ Eppendorf vials for protein assay.

Firstly, we determined the mass loss of studied larvae and materials used. Secondly, we studied biochemical composition of mealworms. Protein content was assessed with the Bradford assay [5]. To determine the level of hydrolized carbohydrates we used anthrone reagent [6] and measure the absorbance of samples at $625 \mathrm{~nm}$. The classical sulfo-phosphovanillin assay (SPVA) was used to quantitate the total lipids in mealworms [7]. Absorbance was measured spectrophotometrically at $525 \mathrm{~nm}$. In all assays multipoint standard curves with high linear regression $(\mathrm{R} 2$ : protein curve $=0.91$; carbohydrate curve $=0.99$; lipids curve $=0.99$ ) were used. All statistical analyses were performed in Statistica ${ }^{\circledR}$ software package. Differences in mass change of mealworms (for five food types) and mass loss for three types of materials with the duration of exposure were tested for each factor using analysis of variance (ANOVA).

\section{Results and discussion}

\subsection{Mass loss measurements}

We determined the percentage of mass changes of larvae and mass loss of used materials after 7, 14 and 21 days from the beginning of the experiment. The results are presented in appropriate Tables (Tab. 1,2) and Figures (Fig. 1).

Table 1. Mass changes of mealworms caused by waste eating and starvation with the comparison of control larvae (g) "_." means decrease of the mass.

\begin{tabular}{|c|c|}
\hline & $\begin{array}{c}\text { Mass changes } \\
\text { of } \boldsymbol{T} \text {. molitor } \\
\text { after 21 days } \\
\text { (\%) }\end{array}$ \\
\hline Control & 45 \\
\hline PS & -18 \\
\hline PLA & -15 \\
\hline PVC & -19 \\
\hline $\begin{array}{c}\text { Starved } \\
\text { larvae }\end{array}$ & -16 \\
\hline
\end{tabular}


Table 2. Mass loss of materials used in studies (g).

\begin{tabular}{|c|c|c|c|}
\hline & $\begin{array}{c}\text { Mass (g) } \\
\text { 1 day }\end{array}$ & $\begin{array}{c}\text { Mass (g) } \\
\mathbf{2 1} \text { day }\end{array}$ & $\begin{array}{c}\text { Mass loss } \\
\text { after 21 days } \\
(\mathbf{\%})\end{array}$ \\
\hline PS & 1,239 & 1,132 & 9 \\
\hline PLA & 0,537 & 0,474 & 12 \\
\hline PVC & 2,052 & 1,995 & 3 \\
\hline
\end{tabular}

The results show the biggest mass change (19\%) for studied larvae fed PVC (Tab. 1, Fig. 1). We also observed the lowest mass loss concerning the mass of PVC (Tab. 2). This is probably due to unavailability of this material to mealworms. PVC for medical purpose was characterised by the smooth surface which make difficult to chew and eat this material by mealworms. They started eating on cutting edges as tubing was served in the form of $10 \mathrm{~mm}$ pieces. Although, we recorded small but noticeable mass loss of PVC (3\%) after 21 days of exposure to mealworms eating, which is interesting and promising in the context of further studies. The masses loss of studied materials were not significantly different $(\mathrm{P}>0.05)$. The significant increasing trend in mass change $(45 \%)$ was observed in the control sample (Tab. 1) which is not surprising. As it was expected, we observed the lowest mass change of larvae for PLA consumption (Table 1) and the highest mass loss of PLA alone (Tab. 2) (Fig. 1). The significant differences were observed only between control mealworms eating oatmeal and larvae fed PVC $(\mathrm{P}<0.05)$. What is suprising, the mass of larvae is greater in starved condition compared to mass of larvae fed on PVC. This is probably due to unavailability and difficulty in chewing of PVC, the larvae lost more energy for make the PVC available for consumption and as a result they lost more mass than starved ones which were waiting for the food without unnecessary energy loss.

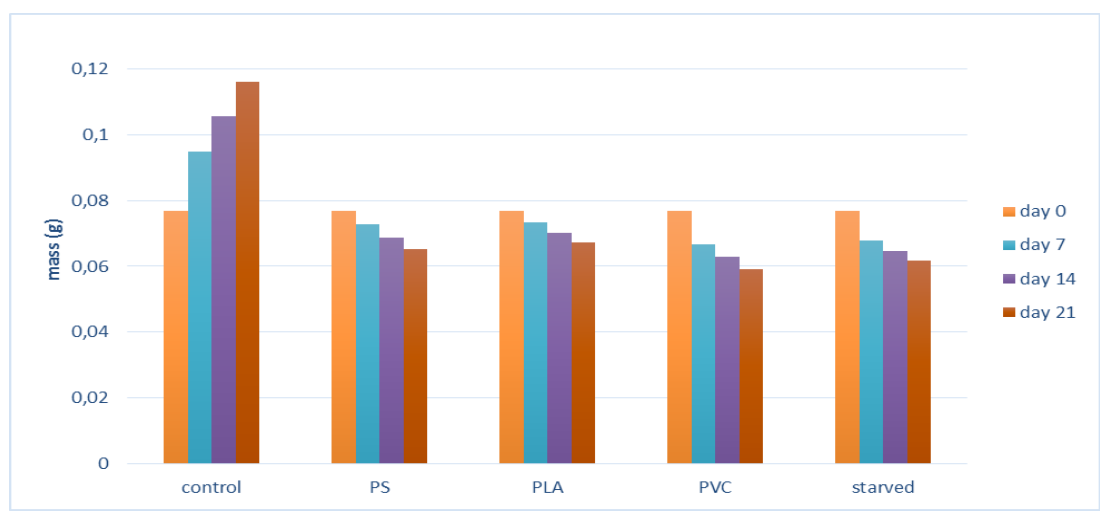

Fig. 1. The average mass of larvae after 7,14 and 21 days of the experiment $(\mathrm{g})$.

\subsection{Biochemical characteristic of mealworms}

\subsubsection{Protein content}

The protein content in the tissues of mealworms not varied significantly depending on the food type. The results are shown in Fig. 2. As the proteins are the main building material for the body of insect they can be used in the hydrolyzed form as fuel for metabolism or converted to glucose through gluconeogenesis. As our results show protein 
levels protein content varied between 16.01-23.38 ng/mg and were not significantly affected by the food type. Some authors noticed that protein synthesis decreases during food deprivation $[8,9]$, leading to a decrease in protein levels as observed here in starved larvae and mealworms fed PS, PVC and PLA. Surprisingly, the highest amount of protein (excluding control) was recorded in larvae fed PLA which may be a result of sparing the energy as a form of adaptation to the difficult conditions and food unavailability. As it was suggested by Renault at al. [8] an insect's recovery from starvation may depend upon its mobility and readiness for searching food, thus a compromise between the demand for carbohydrates and the preservation of muscle proteins is needed. On the other hand, such materials like PVC and PS are not a good source of energy at all which lead to the depletion of proteins in mealworms fed both types of waste.

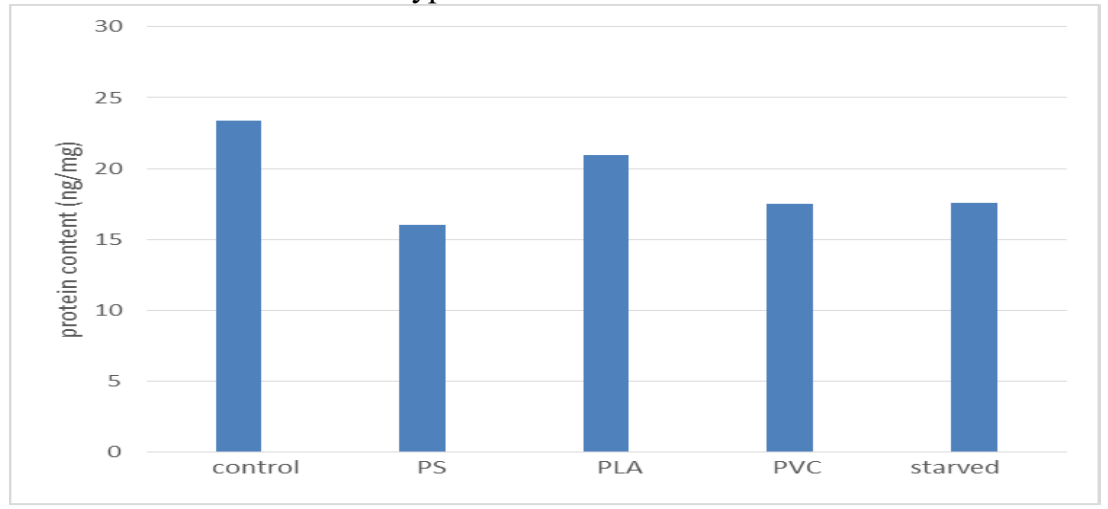

Fig. 2. The protein content in mealworms fed different types of food and starved (ng/mg).

\subsubsection{Carbohydrates content}

Carbohydrates provide most of the energy during insect life. Some insects metabolize glycogen during the initial stages of starvation, then switch to lipid and protein metabolism when carbohydrates are gone. Other species, however, depend more heavily on lipid metabolism during exposition to stress factors [10]. The highest amount of carbohydrates was registered in mealworms fed PLA where the lowest mass change of larvae was observed simultaneously. The lowest carbohydrates content was registered for mealworms fed PVC an PS as these materials are not reliable food source for development and life cycle completion (Fig. 3). As it was shown in the experiment the carbohydrates content was not significantely depleted comparing to the control mealworms fed oatmeal.

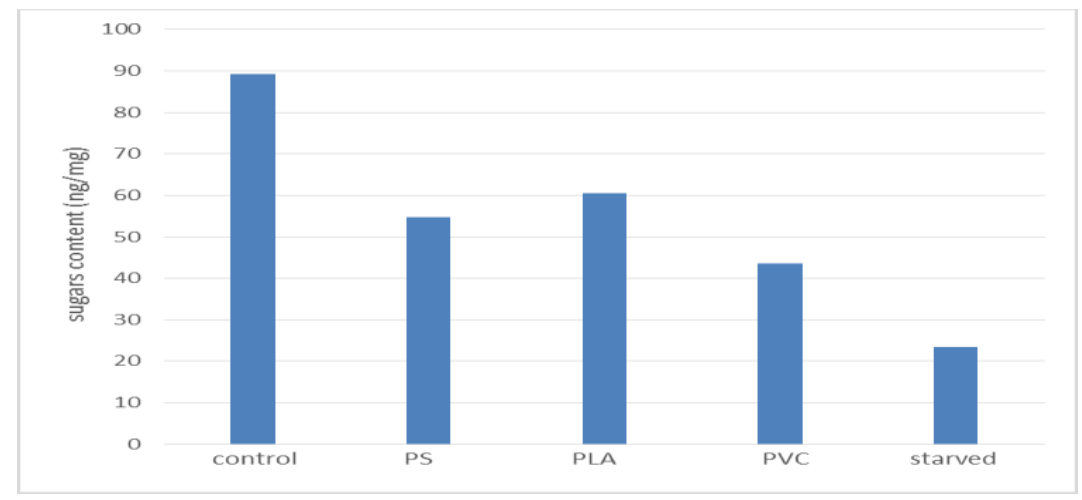

Fig. 3. The carbohydrates content in mealworms fed different types of food and starved (ng/mg). 


\subsubsection{Lipid content}

The most significant changes during stress and starvation are usually observed in the levels of triglycerides, as the level of these compounds decreases significantly. The most common source of energy in insects during starvation period is the oxidation of fatty acids stored in the form of triglyceride, large amounts of lipids are accumulated in insect fat body, with fat content exceeding $50 \%$ of the total dry mass in selected studied insects [10]. Levels of stored fat significantly help to survive during starvation and environmental stress, the level of triglyceride is always connected with the duration of starvation period [10]. In our studies the amount of lipids is the highest in the control mealworms fed oatmeal which is in the line with the observations of other authors suggesting that starvation is connected with the rapid fat reserves burning [10] (Fig. 4). Larvae fed PLA have the highest amount of lipids comparing to mealworms fed PVC and PS which suggest that although they decompose these materials they do not ingest it and use the energy effectively for life activities.

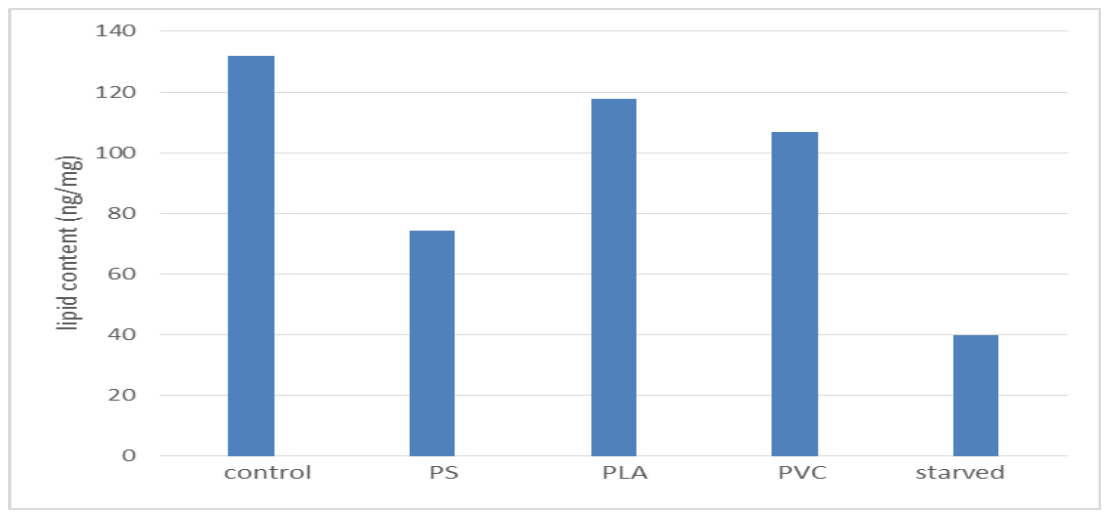

Fig. 4. The lipid content in mealworms fed different types of food and starved (ng/mg).

\section{Conclusions}

In our tests on processing commonly used plastics with $T$. molitor larvae we recorded that all specimens survived three weeks of experiment period, although they required much more effort to maintain their vital functions feeding PS, PVC, PLA or starving. The conducted biochemical assays confirmed that stored energy in the form of lipids and carbohydrates is used for keeping the basic metabolic rates, chewing and eating polymers and depredating them by mealworms. We are aware that the ability of plastics bioprocessing by beetle larvae is strictly connected with the adaptive predisposition of gut microbiome of mealworms. The mass loss of all studied materials (PS, PVC, PLA) suggest that they are eaten by larvae and decomposed efficiently by microorganisms presented in the gut of mealworms. On the other hand, the mass changes of studied specimens indicate that all of these materials are not very efficient source of energy for mealworms, they only allow them to survive but not to deliver a sufficient energy as mealworms lost weight constantly. As it was expected, the lowest mass loss was recorded in the mealworms fed PLA, which is a biodegradable polyester. Although the PVC was not eaten and degraded greatly by mealworms, the obtained results suggest that this material is also biodegradable by bacteria which inhabit the gut of mealworms. Such studies have never been performed yet. 
The interest of scientists in T. molitor larvae is mainly due to their nutritional potential. Additionaly, their ability to process biodegradable materials as well as hardly biodegradable and even non-biodegradable products can be a key feature in further studies and in the future application of these animals for the treatment and disposal of plastic waste which is extremely beneficial for environment health.

\section{References}

1. M. Aruna, V. Shanthi, Int. J. Pharm. Sci. Rev. Res. 36, 5 (2015)

2. J. Maul, B.G. Frushour, J.R. Kontoff, H. Eichenauer, K-H. Ott, C. Schade, Ullmann's Encyclopedia of Industrial Chemistry (Wiley-VCH, Weinheim, 2007)

3. Y. Yang, J. Yang, W-M. Wu, J. Zhao, Y. Song, L. Gao, R. Yang, L. Jiang, Environ. Sci. Technol. 49, 6 (2015)

4. K. M. Nampoothiri, N. R. Nair, R. P. John, Bioresource Technol. 101, 22 (2010)

5. M.M. Bradford, Anal. Biochem. 72, 6 (1976)

6. E. Van Handel, JAMCA 1: 1 (1985)

7. E. Van Handel, JAMCA 1: 2 (1985)

8. D. Renault, F. Hervant, P. Vernon., Physiolog. Entomol. 27, 10 (2002)

9. G. Bosquet, Comp. Biochem. Physiol. 58, 5 (1977)

10. M.T. Marron, T.A. Markow, K.J. Kain, A.G. Gibbs, J. Insect Physiol. 49, 9 (2003) 